\title{
Infinite resonant solutions and turning points in a problem with unbounded bifurcation *
}

\author{
José M. Arrieta ${ }^{1}$, Rosa Pardo $^{1}$, Anibal Rodríguez-Bernal ${ }^{1,2}$ \\ ${ }^{1}$ Departamento de Matemática Aplicada \\ Universidad Complutense de Madrid \\ Madrid 28040, Spain \\ ${ }^{2}$ Instituto de Ciencias Matemáticas \\ CSIC-UAM-UC3M-UCM
}

\begin{abstract}
We consider an elliptic equation $-\Delta u+u=0$ with nonlinear boundary conditions $\frac{\partial u}{\partial n}=\lambda u+g(\lambda, x, u)$, where $\frac{g(\lambda, x, s)}{s} \rightarrow 0$, as $|s| \rightarrow \infty$. In $[1,2]$ the authors proved the existence of unbounded branches of solutions near a Steklov eigenvalue of odd multiplicity and, among other things, provided tools to decide whether the branch is subcritical or supercritical. In this work we give conditions on the nonlinearity guaranteeing the existence of a bifurcating branch which is neither subcritical nor supercritical, having an infinite number of turning points and an infinite number of resonant solutions.
\end{abstract}

Keys words : bifurcation from infinity; nonlinear boundary condtions; Steklov eigenvalues; turning points; resonant solutions.

${ }^{*}$ The three authors are partially supported by Project MTM2006-08262, MEC Spain, GR74/07, Grupo 920894 (Comunidad de Madrid - UCM, Spain). Moreover, the first and third authors are also supported by PHB2006-003PC from MICINN and the first author is also supported by SIMUMAT, Comunidad de Madrid 


\section{Introduction}

In this paper we consider the elliptic problem with nonlinear boundary conditions

$$
\left\{\begin{aligned}
-\Delta u+u & =0, & & \text { in } \Omega \\
\frac{\partial u}{\partial n} & =\lambda u+g(\lambda, x, u), & & \text { on } \partial \Omega .
\end{aligned}\right.
$$

in a bounded and sufficiently smooth domain $\Omega \subset \mathbb{R}^{N}$ with $N \geq 2$.

This problem has already been studied in $[1,2]$ where we analyzed the existence of unbounded sets of solutions as well as their stability and some of the dynamical properties of the associated parabolic problem. This analysis was carried over assuming that the nonlinear term is sublinear at infinity, which roughly speaking means that

$$
|g(\lambda, x, s)|=o(|s|) \quad \text { as }|s| \rightarrow \infty .
$$

This assumption, by a mechanism of parametric resonance at the boundary, produces unbounded branches of solutions when $\lambda$ approaches one of the Steklov eigenvalues of odd multiplicity. These branches bifurcate from infinity in the sense of $[6,5]$. Steklov eigenvalues are the eigenvalues of the eigenvalue problem

$$
\left\{\begin{aligned}
-\Delta \Phi+\Phi & =0, & & \text { in } \Omega \\
\frac{\partial \Phi}{\partial n} & =\sigma \Phi, & & \text { on } \partial \Omega .
\end{aligned}\right.
$$

which form an increasing sequence of real numbers, $\left\{\sigma_{i}\right\}_{i=1}^{\infty}$, ordered and with finite multiplicity, with respective eigenfunctions $\left\{\Phi_{i}\right\}_{i=1}^{\infty}$. The first eigenvalue $\sigma_{1}$ is simple and we can take its eigenfunction $\Phi_{1}$ strictly positive in $\Omega$. Eigenfuntions are orthogonal in $L^{2}(\partial \Omega)$ and $\Phi_{1}$ is normalized in $L^{\infty}(\partial \Omega)$.

The branch bifurcating at $\sigma_{1}$, the first Steklov eigenvalue, forms a continuum in $(\lambda, u) \in$ $\mathbb{R} \times C(\bar{\Omega})$.

The set of solutions bifurcating at $\sigma_{1}$, the first Steklov eigenvalue, is made up of large positive solutions or large negative solutions (or both). We will denote by $\mathcal{D}^{+} \subset \mathbb{R} \times C(\bar{\Omega})$ (resp. $\mathcal{D}^{-} \subset \mathbb{R} \times C(\bar{\Omega})$ ) the branch of positive, (resp. negative) solutions bifurcating at $\sigma_{1}$. As a matter of fact, the solutions in $\mathcal{D}^{ \pm}$, can be described as

$$
u=s \Phi_{1}+w, \quad \text { where } \quad w=o(|s|) \quad \text { as }|s| \rightarrow \infty
$$

see Proposition 3.1, Theorem 3.3 and Theorem 3.4 in [1].

Hereafter we will concentrate on the positive unbounded branch, $\mathcal{D}^{+}$bifurcating at $\sigma_{1}$. The case for $\mathcal{D}^{-}$is completely analogous.

One important question is whether the bifurcating branch $\mathcal{D}^{+}$is subcritical or supercritical. That is, if it is formed only with solutions $(\lambda, u)$ with $\lambda<\sigma_{1}$ or $\lambda>\sigma_{1}$ respectively.

To analyze this question we look at the lower order terms of $g(\lambda, x, s)$ as $\lambda \rightarrow \sigma_{1}$ and $s \rightarrow \infty$. Hence, we define, for $\alpha<1$, the following quantity

$$
\underline{\mathbf{G}}_{+}:=\int_{\partial \Omega} \liminf _{(\lambda, s) \rightarrow\left(\sigma_{1},+\infty\right)} \frac{s g(\lambda, \cdot s)}{|s|^{1+\alpha}} \Phi_{1}^{1+\alpha} .
$$


Changing lim inf by limsup we define the number $\overline{\mathbf{G}}_{+}$. Note that for these numbers to be well defined we are assuming, roughly speaking, that

$$
|g(\lambda, x, s)|=O\left(|s|^{\alpha}\right) \text { as }|s| \rightarrow \infty .
$$

From [1, Theorem 4.3] we know that if $\underline{\mathbf{G}}_{+}>0$, then $\mathcal{D}^{+}$is subcritical, while if $\overline{\mathbf{G}}_{+}<0$, then $\mathcal{D}^{+}$is supercritical.

Another interesting question is that of the resonant problem, that is when $\lambda=\sigma_{1}$. For this case, we obtained in Theorem 5.1 of [1] some Landesman-Lazer type conditions guaranteeing that the resonant problem has solution; see [4]. In the language of bifurcation, these type of conditions can be stated as: if all the unbounded branches are either subcritical or supercritical then the resonant problem has at least one solution.

Therefore, in this paper we consider nonlinearities for which

$$
\underline{\mathbf{G}}_{+}<0<\overline{\mathbf{G}}_{+}
$$

a condition that somehow reflects some oscillatory character of the nonlinear term at infinity, which we hope to translate into an oscilatory behavior of the bifurcating branches. Observe that in this situation, both the criteria for sub/super criticality and the Landesman-Lazer type conditions do not hold.

In such a situation our goal is threefold: first we give easy-to-check conditions on the nonlinear term, guaranteeing that in $\mathcal{D}^{+}$there are large subcritical and supercritical solutions.

Second, the connectedness of $\mathcal{D}^{+}$, suggests that we would be able to find an unbounded sequence of turning points, which are defined as

Definition 1.1 A solution $\left(\lambda^{*}, u^{*}\right)$ of (1.1) in a branch of solutions is called a turning point if there is a neighborhood of $\left(\lambda^{*}, u^{*}\right)$ in $\mathbb{R} \times L^{\infty}(\partial \Omega)$ such that there are no solutions $\left(\lambda, u_{\lambda}\right) \approx$ $\left(\lambda^{*}, u^{*}\right)$, for $\lambda>\lambda^{*}$ or for $\lambda<\lambda^{*}$.

Note that, generically, in a neighborhood of a turning point there are, at least, two solutions for the same value of the parameter at one side, either $\lambda\left\langle\lambda^{*}\right.$, or either for $\lambda>\lambda^{*}$. Therefore, turning points are related with multiplicity of solutions.

Third, the connectedness of $\mathcal{D}^{+}$, suggests that we would be able to find an unbounded sequence of resonant solutions.

A related analysis for the case of an interior reaction term was established in [3] for a one dimensional problem with an interior reaction term. They proved that as the problem approaches resonance, the number of solutions increases to infinity.

This paper is organized as follows. In Section 2 we make precise the hypotheses on the nonlinearity and present in more detail the techniques we use and the main results. In Section 3 we prove the main result of the paper, Theorem 3.4, which gives the existence of unbounded sequences of turning points and resonant solutions. In Section 4 we illustrate our results with two examples, where we consider nonlinear terms of the type

$$
g(x, s):=s^{\alpha}\left[\sin \left(\left|\frac{s}{\Phi_{1}(x)}\right|^{\beta}\right)+C\right]
$$

with $\alpha<1$. 


\section{Preliminaries and description of the results}

With respect to the nonlinearity $g$ in (1.1), we assume the hypotheses

(H1) $g: \mathbb{R} \times \partial \Omega \times \mathbb{R} \rightarrow \mathbb{R}$ is a Carathèodory function (i.e. $g=g(\lambda, x, s)$ is measurable in $x \in \Omega$, and continuous with respect to $(\lambda, s) \in \mathbb{R} \times \mathbb{R})$. Moreover, there exist $h \in L^{r}(\partial \Omega)$ with $r>N-1$ and a continuous functions $\Lambda: \mathbb{R} \rightarrow \mathbb{R}^{+}, U: \mathbb{R} \rightarrow \mathbb{R}^{+}$, satisfying

$$
|g(\lambda, x, s)| \leq \Lambda(\lambda) h(x) U(s), \quad \forall(\lambda, x, s) \in \mathbb{R} \times \partial \Omega \times \mathbb{R} .
$$

Moreover, we assume the following condition on the function $U$

(H2) $\lim _{|s| \rightarrow \infty} \frac{U(s)}{s}=0$

which in turn it implies that $\lim \sup _{|s| \rightarrow \infty}|g(\lambda, x, s) / s| \rightarrow 0$, that is, the function $g$ is sublinear at infinity in the variable $s$.

(H3) The nonlinearity $g(\lambda, x, s)$ is differentiable in $s$ and

$$
\frac{\partial g}{\partial s}(\lambda, \cdot, \cdot) \in C(\partial \Omega \times \mathbb{R})
$$

Note that, as in [1, 2], solutions of (1.1) are determined and estimated in terms of their boundary values. Therefore, we can look at (1.1) as a problem posed in a space of functions defined on $\partial \Omega$.

Now we describe the technique we follow to prove the main result, Theorem 3.4. Note that this result gives easy-to-check conditions on the nonlinear term, guaranteeing that in $\mathcal{D}^{+}$there are large subcritical and supercritical solutions. We start out of (1.2), from where we know that for $(\lambda, u) \in \mathcal{D}^{+}$with $\lambda \rightarrow \sigma_{1}$ we have

$$
u=s \Phi_{1}+w, \quad \text { where } w=o(s), \quad \int_{\partial \Omega} w \Phi_{1}=0 \quad \text { as } s \rightarrow \infty .
$$

With this, we are able to prove that if

$$
|g(\lambda, x, s)|=O\left(|s|^{\alpha}\right) \quad \text { as } \quad s \rightarrow \infty
$$

then

$$
w=O\left(|s|^{\alpha}\right) \quad \text { as } \quad s \rightarrow \infty, \quad \text { and } \quad\left|\sigma_{1}-\lambda\right|=O\left(|s|^{\alpha-1}\right) \quad \text { as } \quad s \rightarrow \infty
$$

see Proposition 3.2. 
Now, consider a sequence $\left(\lambda_{n}, u_{n}\right) \in \mathcal{D}^{+}$with $\lambda_{n} \rightarrow \sigma_{1}$ and $\left\|u_{n}\right\|_{L^{\infty}(\partial \Omega)} \rightarrow \infty$. Using the results in [1], to determine whether a sequence of solutions lies at one side or another of $\sigma_{1}$ one must check the signs of

$$
\liminf _{n \rightarrow \infty} \int_{\partial \Omega} \frac{u_{n} g\left(\lambda_{n}, \cdot, u_{n}\right)}{\left|u_{n}\right|^{1+\alpha}} \Phi_{1}^{1+\alpha}, \quad \limsup _{n \rightarrow \infty} \int_{\partial \Omega} \frac{u_{n} g\left(\lambda_{n}, \cdot, u_{n}\right)}{\left|u_{n}\right|^{1+\alpha}} \Phi_{1}^{1+\alpha},
$$

see also [2, Lemma 3.1]. But this requires a knowledge of the solutions themselves.

Using the previous results, we write

$$
u_{n}=s_{n} \Phi_{1}+w_{n}, \quad \text { where } w_{n}=O\left(\left|s_{n}\right|^{\alpha}\right), \quad \int_{\partial \Omega} w_{n} \Phi_{1}=0 \quad \text { as } n \rightarrow \infty,
$$

and we intend to unveil the signs in (2.1) by just looking at the signs of

$$
\liminf _{n \rightarrow \infty} \int_{\partial \Omega} \frac{s_{n} g\left(\sigma_{1}, \cdot, s_{n} \Phi_{1}\right)}{\left|s_{n}\right|^{1+\alpha}} \Phi_{1}, \quad \limsup _{s_{n} \rightarrow \infty} \int_{\partial \Omega} \frac{s_{n} g\left(\sigma_{1}, \cdot, s_{n} \Phi_{1}\right)}{\left|s_{n}\right|^{1+\alpha}} \Phi_{1} .
$$

This is achieved in Lemma 3.3.

With these tools, in Theorem 3.4 we take two sequences $\left\{s_{n}\right\}$ and $\left\{s_{n}^{\prime}\right\}$ satisfying

$$
0<\lim _{n \rightarrow+\infty} \int_{\partial \Omega} s_{n} \frac{g\left(\sigma_{1}, \cdot, s_{n} \Phi_{1}\right)}{\left|s_{n}\right|^{1+\alpha}} \Phi_{1}<\infty, \quad-\infty<\lim _{n \rightarrow+\infty} \int_{\partial \Omega} \frac{s_{n}^{\prime} g\left(\sigma_{1}, \cdot, s_{n}^{\prime} \Phi_{1}\right)}{\left|s_{n}^{\prime}\right|^{1+\alpha}} \Phi_{1}<0
$$

and from here we obtain the existence of unbounded sequences of sub and supercritical solutions of $(1.1)$ in $\mathcal{D}^{+}$.

Finally exploiting the connectedness of $\mathcal{D}^{+}$, we obtain the existence of unbounded sequences of turning points and of resonant solutions.

\section{Multiple turning points and resonant solutions}

In this section we give sufficient conditions for the existence of a branch of solutions bifurcating from infinity which is neither subcritical nor supercritical. From this, we conclude the existence of infinitely many turning points, see Definition 1.1, and an infinite number of solutions for the resonant problem, i.e. for $\lambda=\sigma_{1}$. This is achieved in Theorem 3.4

For this we first consider a family of linear Steklov problems with a variable nonhomogeneous term at the boundary $h$ depending on the parameter $\lambda$

$$
\left\{\begin{aligned}
-\Delta u+u & =0, & & \text { in } \Omega \\
\frac{\partial u}{\partial n} & =\lambda u+h(\lambda, x), & & \text { on } \partial \Omega
\end{aligned}\right.
$$

where $h(\lambda, \cdot) \in L^{r}(\partial \Omega), r>N-1$ and $\lambda \in\left(-\infty, \sigma_{2}\right)$.

We use now the decomposition

$$
L^{r}(\partial \Omega)=\operatorname{span}\left[\Phi_{1}\right] \oplus \operatorname{span}\left[\Phi_{1}\right]^{\perp}, \quad \text { where } \quad \operatorname{span}\left[\Phi_{1}\right]^{\perp}:=\left\{u \in L^{r}(\partial \Omega): \int_{\partial \Omega} u \Phi_{1}=0\right\}
$$


and then for $h(\lambda, \cdot) \in L^{r}(\partial \Omega)$, with $r>N-1$, there exists a unique decomposition

$$
h(\lambda, \cdot)=a_{1}(\lambda) \Phi_{1}+h_{1}(\lambda, \cdot), \quad \text { where } \quad a_{1}(\lambda)=\frac{\int_{\partial \Omega} h(\lambda, \cdot) \Phi_{1}}{\int_{\partial \Omega} \Phi_{1}^{2}}, \quad \text { and } \quad \int_{\partial \Omega} h_{1}(\lambda, \cdot) \Phi_{1}=0 .
$$

The Fredholm Alternative states that the linear problem (3.1) has a unique solution if $\lambda \neq \sigma_{1}$ and does not have solution if $\lambda=\sigma_{1}$ and $a_{1}\left(\sigma_{1}\right)=\frac{\int_{\partial \Omega} h\left(\sigma_{1}, \cdot\right) \Phi_{1}}{\int_{\partial \Omega} \Phi_{1}{ }^{2}} \neq 0$.

Hence, for $\lambda \neq \sigma_{1}$ the solution $u=u(\lambda)$ of (3.1) has a unique decomposition

$$
u=\frac{a_{1}(\lambda)}{\sigma_{1}-\lambda} \Phi_{1}+w, \quad \text { where } \quad \int_{\partial \Omega} w \Phi_{1}=0,
$$

and $w=w(\lambda) \in \operatorname{span}\left[\Phi_{1}\right]^{\perp}$ solves the problem

$$
\left\{\begin{aligned}
-\Delta w+w & =0, & & \text { in } \Omega \\
\frac{\partial w}{\partial n} & =\lambda w+h_{1}(\lambda, x), & & \text { on } \partial \Omega .
\end{aligned}\right.
$$

Note that in (3.4) $w(\lambda) \in \operatorname{span}\left[\Phi_{1}\right]^{\perp}$ is also well defined for $\lambda=\sigma_{1}$.

The next result states that $w=w(\lambda)$ is uniformly bounded if $h(\lambda, \cdot)$ is so.

Lemma 3.1 For each compact set $K \subset\left(-\infty, \sigma_{2}\right) \subset \mathbb{R}$ there exists a constant $C=C(K)$, independent of $\lambda$, such that

$$
\|w(\lambda)\|_{L^{\infty}(\partial \Omega)} \leq C\|h(\lambda, \cdot)\|_{L^{r}(\partial \Omega)} \quad \text { for any } \quad \lambda \in K .
$$

where $w \in \operatorname{span}\left[\Phi_{1}\right]^{\perp}$ is the solution of (3.4) and $h_{1} \in \operatorname{span}\left[\Phi_{1}\right]^{\perp}$ is defined in (3.2).

Proof. Note again that by the Fredholm Alternative the solution of (3.4), $w=w(\lambda) \in \operatorname{span}\left[\Phi_{1}\right]^{\perp}$ is well defined for any $\lambda \in K$.

First, we prove that $w(\lambda)$ is uniformly bounded for any $\lambda$ in a neighborhood of $\sigma_{1}$. Assume this is not the case. Then, there is a sequence $\lambda_{n} \rightarrow \sigma_{1}$ with $\left\|w\left(\lambda_{n}\right)\right\|_{L^{\infty}(\partial \Omega)} \rightarrow \infty$. From [1, Corollary 3.2], we have $\frac{w\left(\lambda_{n}\right)}{\left\|w\left(\lambda_{n}\right)\right\|_{L^{\infty}(\partial \Omega)}} \rightarrow \Phi_{1}$ uniformly in $\bar{\Omega}$, contradicting the fact that $w(\lambda) \in$ $\operatorname{span}\left[\Phi_{1}\right]^{\perp}$. Therefore, there exists some $\delta>0$ such that $\|w(\lambda)\|_{L^{\infty}}<c$ independent of $\lambda$ for any $\left|\lambda-\sigma_{1}\right|<\delta$.

Second, $\|w(\lambda)\|_{L^{\infty}}<\infty$ for any $\lambda \in K \backslash\left(\sigma_{1}-\delta, \sigma_{1}+\delta\right)$, since the linear operator is invertible; see Theorem 2.7 in [1].

Now we define the family of operators $T(\lambda): L^{r}(\partial \Omega) \rightarrow L^{\infty}(\partial \Omega)$ by $T(\lambda) h:=w(\lambda)$. Then $T(\lambda)$ is continuous for every $\lambda \in K$ and $\sup _{\lambda \in K}\|T(\lambda) h\|_{L^{\infty}(\partial \Omega)}<\infty$. Therefore, applying the uniform boundedness principle, there exists a constant $C=C(K)$ such that

$$
\|w(\lambda)\|_{L^{\infty}(\partial \Omega)} \leq C(K)\|h\|_{L^{r}(\partial \Omega)} \quad \text { for any } \quad \lambda \in K
$$

and we get the result. $\square$ 
Now we turn into the nonlinear problem (1.1). Recall that for $\lambda$ close to $\sigma_{1}$ we have (1.2), that is, as $\lambda \rightarrow \sigma_{1}$ the unbounded solutions satisfy

$$
u=s \Phi_{1}+w, \quad \text { where } w=o(s), \quad w \in \operatorname{span}\left[\Phi_{1}\right]^{\perp} \quad \text { as } s \rightarrow \infty .
$$

For later use, we define

$$
P(u)=\frac{\int_{\partial \Omega} u(\cdot) \Phi_{1}}{\int_{\partial \Omega} \Phi_{1}^{2}} .
$$

Then, we give conditions on the nonlinear term $g$ in (1.1), guaranteeing that in (3.5) the order of $w$ in (3.5) is $w=O\left(|s|^{\alpha}\right)$ as $s \rightarrow \infty$. Note that we restrict ourselves below to the unbounded branch of positive solutions. A completely analogous result holds for the unbounded branch of negative solutions.

Proposition 3.2 Assume g satisfies hypotheses (H1) and (H2). We also assume that for some $\alpha<1$ there exists a function $G_{1}$ such that for $\lambda \rightarrow \sigma_{1}$, for sufficiently large $s>0$ and $x \in \partial \Omega$ we have

$$
\frac{|g(\lambda, x, s)|}{|s|^{\alpha}} \leq G_{1}(x), \quad G_{1} \in L^{r}(\partial \Omega), \quad r>N-1 .
$$

Then, there exists an open set $\mathcal{O} \subset \mathbb{R} \times C(\bar{\Omega})$ of the form $\mathcal{O}=\left\{(\lambda, u):\left|\lambda-\sigma_{1}\right|<\delta_{0}, u(x)>\right.$ $\left.M_{0}\right\}$ for some small $\delta_{0}$ and large $M_{0}$, such that $\mathcal{D}^{+}$, the unbounded branch of positive solutions of (1.1), satisfies

(i) There exists a constant $C_{1}$ independent of $\lambda$ such that, if $(\lambda, u) \in \mathcal{D}^{+} \cap \mathcal{O}$ and $(\lambda, u) \neq$ $\left(\sigma_{1}, \infty\right)$ then $u=s \Phi_{1}+w$, where $s>0, w \in \operatorname{span}\left[\Phi_{1}\right]^{\perp}$ and

$$
\|w\|_{L^{\infty}(\partial \Omega)} \leq C_{1}\left\|G_{1}\right\|_{L^{r}(\partial \Omega)}|s|^{\alpha}, \quad \text { as }|s| \rightarrow \infty
$$

(ii) There exists some constant $S_{0}>0$ such that for all $s \geq S_{0}$ there exists a solution $(\lambda, u) \in \mathcal{D}^{+} \cap \mathcal{O}$ satisfying $u=s \Phi_{1}+w$, with $w \in \operatorname{span}\left[\Phi_{1}\right]^{\perp}$.

(iii) Moreover, there exists a constant $C_{2}$ independent of $\lambda$ such that, for any solution of the type $(\lambda, u) \in \mathcal{D}^{+} \cap \mathcal{O}, u=s \Phi_{1}+w$, with $w \in \operatorname{span}\left[\Phi_{1}\right]^{\perp}$, the following holds

$$
\left|\sigma_{1}-\lambda\right| \leq C_{2}|s|^{\alpha-1}, \quad \text { as }|s| \rightarrow \infty, \quad \text { with } C_{2}=\frac{2\left\|G_{1}\right\|_{L^{1}(\partial \Omega)}}{\int_{\partial \Omega} \Phi_{1}^{2}} .
$$

Proof. Note that (3.7), Lemma 3.1 and the fact that, from (3.5), $\Phi_{1}+w / s \rightarrow \Phi_{1}$ as $s \rightarrow \infty$ in $L^{\infty}(\partial \Omega)$ imply that in fact that $\|w\|_{L^{\infty}(\partial \Omega)} \leq C|s|^{\alpha}$ as $s \rightarrow \infty$. This proves part $\left.i\right)$.

To prove part ii) note that $\mathcal{D}^{+} \cap \mathcal{O}$, although not necessarily connected, it has an unbounded connected component. Hence, using the decomposition (3.5), we have $u=s \Phi_{1}+w$ with $w \in$ $\operatorname{span}\left[\Phi_{1}\right]^{\perp}$. Since the projection (3.6) is continuous, the set $s \in \mathbb{R}$ such that there exists a solution of (1.1) with $u=s \Phi_{1}+w$ with $w \in \operatorname{span}\left[\Phi_{1}\right]^{\perp}$ contains an unbounded connected set in $\mathbb{R}$. 
To prove part $i i i)$, we observe that if $(\lambda, u)$ is a solution of (1.1), $u=s \Phi_{1}+w$, with $w \in$ $\operatorname{span}\left[\Phi_{1}\right]^{\perp}$, multiplying the equation by the first Steklov eigenfunction $\Phi_{1}>0$ and integrating by parts we obtain,

$$
\left(\sigma_{1}-\lambda\right) s \int_{\partial \Omega} \Phi_{1}^{2}=\int_{\partial \Omega} g\left(\lambda, x, s \Phi_{1}+w\right) \Phi_{1}
$$

Taking into account that

$$
\frac{\left|g\left(\lambda, x, s \Phi_{1}+w\right)\right|}{|s|}=\frac{\left|g\left(\lambda, x, s \Phi_{1}+w\right)\right|}{\left|s \Phi_{1}+w\right|}\left|\Phi_{1}+\frac{w}{s}\right| \rightarrow 0, \quad \text { as } \quad s \rightarrow \infty
$$

we get $\lambda \rightarrow \sigma_{1}$ as $s \rightarrow \infty$.

Moreover, from (3.7), we obtain that

$$
\left|g\left(\lambda, x, s \Phi_{1}+w\right)\right|=|s|^{\alpha} \frac{\left|g\left(\lambda, x, s \Phi_{1}+w\right)\right|}{\left|s \Phi_{1}+w\right|^{\alpha}}\left|\Phi_{1}+\frac{w}{s}\right|^{\alpha} \leq|s|^{\alpha} G_{1}(x)\left|\Phi_{1}+\frac{w}{s}\right|^{\alpha}
$$

and therefore

$$
\left|\sigma_{1}-\lambda\right| \leq \frac{|s|^{\alpha-1}}{\int_{\partial \Omega} \Phi_{1}^{2}} \int_{\partial \Omega} G_{1}(x)\left|\Phi_{1}+\frac{w}{s}\right|^{\alpha} \Phi_{1} \leq C\left\|G_{1}\right\|_{L^{r}(\partial \Omega)}|s|^{\alpha-1}
$$

which ends the proof.

After this, in order to prove the main result, Theorem 3.4 below, we need to guarantee that the signs in (2.1) can be determined by the signs in (2.2), that is

$$
\liminf _{(\lambda, s) \rightarrow\left(\sigma_{1},+\infty\right)} \int_{\partial \Omega} \frac{s g\left(\lambda, \cdot, s \Phi_{1}\right)}{|s|^{1+\alpha}} \Phi_{1}<0<\limsup _{(\lambda, s) \rightarrow\left(\sigma_{1},+\infty\right)} \int_{\partial \Omega} \frac{s g\left(\lambda, \cdot s \Phi_{1}\right)}{|s|^{1+\alpha}} \Phi_{1} .
$$

In order to guarantee that (3.8) is enough to conclude the existence of sub and supercitical solution in the unbounded branch, we will use the following result.

Lemma 3.3 Given $h(\lambda, x, s)$, differentiable with respect to the last variable, assume that for some $\alpha<1$ there exists a function $H_{1}$ such that for all $(\lambda, s) \approx\left(\sigma_{1},+\infty\right)$ and $x \in \partial \Omega$ we have

$$
\left|\frac{h(\lambda, x, s)}{|s|^{\alpha}}\right| \leq H_{1}(x), \quad H_{1} \in L^{1}(\partial \Omega)
$$

Assume also its partial derivative $\frac{\partial h}{\partial s}(\lambda, \cdot, \cdot) \in C(\partial \Omega \times \mathbb{R})$, for any $\lambda \approx \sigma_{1}$, and

$$
\sup _{|s| \geq M}\left\|\frac{\partial h}{\partial s}(\lambda, \cdot, s)\right\|_{L^{\infty}(\partial \Omega)} \rightarrow 0 \quad \text { as } \quad \lambda \rightarrow \sigma_{1}, \quad M \rightarrow+\infty
$$

Let $\lambda_{n} \rightarrow \sigma_{1}, s_{n} \uparrow \infty$ and $w_{n}$ in $L^{\infty}(\partial \Omega)$, such that $\left\|w_{n}\right\|_{L^{\infty}(\partial \Omega)} \leq C\left|s_{n}\right|^{\alpha}$ as $n \rightarrow \infty$ for some constant $C$. Then the following holds

$$
\liminf _{n \rightarrow+\infty} \int_{\partial \Omega} \frac{\left(s_{n} \Phi_{1}+w_{n}\right) h\left(\lambda_{n}, \cdot, s_{n} \Phi_{1}+w_{n}\right)}{\left|s_{n} \Phi_{1}+w_{n}\right|^{1+\alpha}} \Phi_{1}^{1+\alpha} \geq \liminf _{n \rightarrow+\infty} \int_{\partial \Omega} \frac{\operatorname{sh}\left(\lambda_{n}, \cdot, s_{n} \Phi_{1}\right)}{\left|s_{n}\right|^{1+\alpha}} \Phi_{1},
$$


and similarly

$$
\limsup _{n \rightarrow+\infty} \int_{\partial \Omega} \frac{\left(s_{n} \Phi_{1}+w_{n}\right) h\left(\lambda_{n}, \cdot, s_{n} \Phi_{1}+w_{n}\right)}{\left|s_{n} \Phi_{1}+w_{n}\right|^{1+\alpha}} \Phi_{1}^{1+\alpha} \leq \limsup _{n \rightarrow+\infty} \int_{\partial \Omega} \frac{\operatorname{sh}\left(\lambda_{n}, \cdot, s_{n} \Phi_{1}\right)}{\left|s_{n}\right|^{1+\alpha}} \Phi_{1} .
$$

Proof. For all $(\lambda, s) \approx\left(\sigma_{1},+\infty\right)$ and for any $w \in L^{\infty}(\partial \Omega)$ such that $\frac{1}{2} \Phi_{1}>\frac{|w|}{s}$, we have (with a constant $C$ that may change from line to line)

$$
\begin{aligned}
\int_{\partial \Omega}\left|h\left(\lambda, \cdot, s \Phi_{1}+w\right)-h\left(\lambda, \cdot, s \Phi_{1}\right)\right| \Phi_{1} & \leq C\|w\|_{L^{\infty}(\partial \Omega)} \int_{\partial \Omega}\left|\int_{0}^{1} \frac{\partial h}{\partial s}\left(\lambda, \cdot, s \Phi_{1}+\tau w\right) d \tau\right| \\
& \leq C\|w\|_{L^{\infty}(\partial \Omega)} \sup _{\tau \in[0,1]}\left\|\frac{\partial h}{\partial s}\left(\lambda, \cdot, s \Phi_{1}+\tau w\right)\right\|_{L^{\infty}(\partial \Omega)}
\end{aligned} .
$$

Taking into account hypothesis (3.10) and whenever $\|w\|_{L^{\infty}(\partial \Omega)}=O\left(|s|^{\alpha}\right)$, we deduce that

$$
\begin{aligned}
\int_{\partial \Omega} \frac{\left|h\left(\lambda, \cdot, s \Phi_{1}+w\right)-h\left(\lambda, \cdot s \Phi_{1}\right)\right|}{|s|^{\alpha}} \Phi_{1} \\
\leq C \sup _{|s| \geq M}\left\|\frac{\partial h}{\partial s}(\lambda, \cdot, s)\right\|_{L^{\infty}(\partial \Omega)} \longrightarrow 0 \text { as } \lambda \rightarrow \sigma_{1}, M \rightarrow+\infty .
\end{aligned}
$$

Consequently, for $\left\|w_{n}\right\|_{L^{\infty}(\partial \Omega)}=O\left(\left|s_{n}\right|^{\alpha}\right)$

$$
\begin{aligned}
\liminf _{n \rightarrow+\infty} \int_{\partial \Omega} \frac{s_{n} h\left(\lambda_{n}, \cdot, s_{n} \Phi_{1}+w_{n}\right)}{\left|s_{n}\right|^{1+\alpha}} \Phi_{1} \geq & \lim _{\substack{\lambda \rightarrow \sigma_{1} \\
s \rightarrow+\infty}} \int_{\partial \Omega} \frac{s h\left(\lambda, \cdot s \Phi_{1}+w\right)-\operatorname{sh}\left(\lambda, \cdot s \Phi_{1}\right)}{|s|^{1+\alpha}} \Phi_{1} \\
& +\liminf _{n \rightarrow+\infty} \int_{\partial \Omega} \frac{s_{n} h\left(\lambda_{n}, \cdot, s_{n} \Phi_{1}\right)}{\left|s_{n}\right|^{1+\alpha}} \Phi_{1} \\
= & \liminf _{n \rightarrow+\infty} \int_{\partial \Omega} \frac{s_{n} h\left(\lambda_{n}, \cdot, s_{n} \Phi_{1}\right)}{\left|s_{n}\right|^{1+\alpha}} \Phi_{1},
\end{aligned}
$$

where we used (3.11).

Now note that the left hand side above can be written as

$$
\frac{s_{n} h\left(\lambda_{n}, \cdot, s_{n} \Phi_{1}+w_{n}\right)}{\left|s_{n}\right|^{1+\alpha}} \Phi_{1}=\frac{\left(s_{n} \Phi_{1}+w_{n}\right) h\left(\lambda_{n}, \cdot s_{n} \Phi_{1}+w_{n}\right)}{\left|s_{n} \Phi_{1}+w_{n}\right|^{1+\alpha}}\left|\Phi_{1}+\frac{w_{n}}{s_{n}}\right|^{\alpha} \Phi_{1} .
$$

Then, (3.9) and the fact that $\Phi_{1}+w_{n} / s_{n} \rightarrow \Phi_{1}$ in $L^{\infty}(\partial \Omega)$ conclude the proof. $\square$

Now we are in a position to prove the main result in this paper that, roughly speaking, states that if there are a sequence of subcritical solutions and another of supercritical solutions, since the solution set is connected, there are infinite turning points and infinite resonant solutions. We state the result for the positive branch. The same conclusions can be attained for the connected branch of negative solutions bifurcating from infinity. 
Theorem 3.4 Assume the nonlinearity g satisfies hypothesis (H1), (H2) and (H3). Assume that for some $\alpha<1$ there exists a function $G_{1}$ such that for for $\lambda \rightarrow \sigma_{1}$, for sufficiently large $s>0$ and $x \in \partial \Omega$ we have

$$
\frac{|g(\lambda, x, s)|}{|s|^{\alpha}} \leq G_{1}(x), \quad G_{1} \in L^{r}(\partial \Omega), \quad r>N-1 .
$$

Assume also that

$$
\sup _{|s| \geq M}\left\|\frac{\partial g}{\partial s}(\lambda, \cdot, s)\right\|_{L^{\infty}(\partial \Omega)} \rightarrow 0 \quad \text { as } \quad \lambda \rightarrow \sigma_{1}, M \rightarrow+\infty
$$

and that

$$
\sup _{|s| \geq M}\left|\frac{g(\lambda, x, s)-g\left(\sigma_{1}, x, s\right)}{|s|^{\alpha}}\right| \rightarrow 0 \quad \text { as } \quad \lambda \rightarrow \sigma_{1}, M \rightarrow+\infty
$$

pointwise in $x$.

Assume moreover that there exist two increasing sequences $\left\{s_{n}\right\},\left\{s_{n}^{\prime}\right\}$ both convergent to $+\infty$, such that

$$
0<\lim _{n \rightarrow+\infty} \int_{\partial \Omega} s_{n} \frac{g\left(\sigma_{1}, \cdot, s_{n} \Phi_{1}\right)}{\left|s_{n}\right|^{1+\alpha}} \Phi_{1}<\infty, \quad-\infty<\lim _{n \rightarrow+\infty} \int_{\partial \Omega} s_{n}^{\prime} \frac{g\left(\sigma_{1}, \cdot, s_{n}^{\prime} \Phi_{1}\right)}{\left|s_{n}^{\prime}\right|^{1+\alpha}} \Phi_{1}<0
$$

Then, in the connected branch of positive solutions bifurcating from infinity, $\mathcal{D}^{+}$, the following assertions hold.

i) For sufficiently large $n \gg 1$, any solution $(\lambda, u)$ is subcritical if

$$
P(u)=\frac{\int_{\partial \Omega} u \Phi_{1}}{\int_{\partial \Omega} \Phi_{1}^{2}}=s_{n}
$$

and supercritical if $P(u)=s_{n}^{\prime}$. Consequently, there exist two sequences of solutions of (1.1), $\left\{\left(\lambda_{n}, u_{n}\right)\right\}$ and $\left\{\left(\lambda_{n}^{\prime}, u_{n}^{\prime}\right)\right\}$ converging to $\left(\sigma_{1}, \infty\right)$ as $n \rightarrow \infty$, one of them subcritical, $\lambda_{n}<\sigma_{1}$, and the other supercritical, $\lambda_{n}^{\prime}>\sigma_{1}$.

ii) There is an unbounded sequence of turning points $\left\{\left(\lambda_{n}^{*}, u_{n}^{*}\right)\right\}$ such that

$$
\lambda_{n}^{*} \rightarrow \sigma_{1}, \quad\left\|u_{n}^{*}\right\|_{L^{\infty}(\partial \Omega)} \rightarrow \infty, \quad \text { as } n \rightarrow \infty .
$$

In fact, we can always choose two subsequences of turning points, one of them subcritical, $\lambda_{2 n+1}^{*}<\sigma_{1}$, and the other supercritical, $\lambda_{2 n}^{*}>\sigma_{1}$.

iii) There is an unbounded sequence of resonant solutions, i.e. there are infinite solutions $\left\{\left(\sigma_{1}, \widetilde{u}_{n}\right)\right\}$ of $(1.1)$ with $\left\|\widetilde{u}_{n}\right\|_{L^{\infty}(\partial \Omega)} \rightarrow \infty$. 
Proof. From Proposition 3.2, ii), consider any two sequences of solutions of (1.1), such that $\left(\lambda_{n}, u_{n}\right) \rightarrow\left(\sigma_{1}, \infty\right)$ and $\left(\lambda_{n}^{\prime}, u_{n}^{\prime}\right) \rightarrow\left(\sigma_{1}, \infty\right)$ in $\mathcal{D}^{+}$with

$$
P\left(u_{n}\right)=\frac{\int_{\partial \Omega} u_{n} \Phi_{1}}{\int_{\partial \Omega} \Phi_{1}^{2}}=s_{n}, \quad \text { and } \quad P\left(u_{n}^{\prime}\right)=\frac{\int_{\partial \Omega} u_{n}^{\prime} \Phi_{1}}{\int_{\partial \Omega} \Phi_{1}^{2}}=s_{n}^{\prime} .
$$

Writing $u_{n}=s_{n} \Phi_{1}+w_{n}$, with $w_{n} \in \operatorname{span}\left[\Phi_{1}\right]^{\perp}$, from Proposition $\left.3.2 \mathrm{i}\right)$, we have $\left\|w_{n}\right\|_{L^{\infty}(\partial \Omega)}=$ $O\left(\left|s_{n}\right|^{\alpha}\right)$. Now, from [2, Lemma 3.1], hypotheses (3.12)-(3.13), Lemma 3.3, and hypotheses (3.14) and (3.15) we get that

$$
\begin{aligned}
\liminf _{n \rightarrow \infty} \frac{\sigma_{1}-\lambda_{n}}{\left\|u_{n}\right\|_{L^{\infty}(\partial \Omega)}^{\alpha-1}} & \geq \frac{1}{\int_{\partial \Omega} \Phi_{1}^{2}} \liminf _{n \rightarrow \infty} \int_{\partial \Omega} \frac{\left(s_{n} \Phi_{1}+w_{n}\right) g\left(\lambda_{n}, \cdot s_{n} \Phi_{1}+w_{n}\right)}{\left|s_{n} \Phi_{1}+w_{n}\right|^{1+\alpha}} \Phi_{1}^{1+\alpha} \\
& \geq \frac{1}{\int_{\partial \Omega} \Phi_{1}^{2}} \liminf _{n \rightarrow+\infty} \int_{\partial \Omega} \frac{s_{n} g\left(\lambda_{n}, \cdot, s_{n} \Phi_{1}\right)}{\left|s_{n}\right|^{1+\alpha}} \Phi_{1} \\
& =\frac{1}{\int_{\partial \Omega} \Phi_{1}^{2}} \liminf _{n \rightarrow+\infty} \int_{\partial \Omega} \frac{s_{n} g\left(\sigma_{1}, \cdot, s_{n} \Phi_{1}\right)}{\left|s_{n}\right|^{1+\alpha}} \Phi_{1}>0,
\end{aligned}
$$

and therefore $\lambda_{n}<\sigma_{1}$.

Analogously, for $\left(\lambda_{n}^{\prime}, u_{n}^{\prime}\right)$ we get $\lambda_{n}^{\prime}>\sigma_{1}$. Hence i) is proved.

To prove ii), assume, by choosing subsequences if necessary, that $s_{n}<s_{n}^{\prime}<s_{n+1}$ for all $n \geq 0$ and that $s_{n}, s_{n}^{\prime} \geq S_{0}$ where $S_{0}$ is the one from Proposition 3.2 ii). In particular, from i) and ii) of Proposition 3.2 we have that if $(\lambda, u) \in \mathcal{D}^{+}$and $P(u)=s>S_{0}$ then $\|u\|_{L^{\infty}(\partial \Omega)} \leq$ $\left(1+C_{1}\left\|G_{1}\right\|_{L^{r}(\partial \Omega)}\left|S_{0}\right|^{\alpha-1}\right) s$. Again, taking $S_{0}$ large enough we can assume $\|u\|_{L^{\infty}(\partial \Omega)} \leq 2 s$.

Define the set

$$
K_{n}=\left\{(\lambda, u) \in \mathcal{D}^{+}, \text {with } P(u)=s, \text { and } s_{n} \leq s \leq s_{n+1}\right\}
$$

Let us show that, for each $n \in \mathbb{N}, K_{n}$ is a compact set in $\mathbb{R} \times C(\bar{\Omega})$.

Let us take a sequence in $\left(\mu_{k}, v_{k}\right) \in K_{n}$ and let us extract a subsequence, that we also denote by $\left(\mu_{k}, v_{k}\right)$ with the property that $\mu_{k} \rightarrow \mu^{*}$. Obviously $s_{n} \leq P\left(v_{k}\right) \leq s_{n+1}$ for all $k$ which implies the bounds $\left\|v_{k}\right\|_{C(\partial \Omega)} \leq 2 s_{n+1}$ for all $k$.

Using these a priori bounds on the solutions we have, see [1, Proposition 2.3],

$$
\left\|v_{k}\right\|_{C^{\alpha}(\bar{\Omega})} \leq C_{1}\left(1+\left\|v_{k}\right\|_{L^{\infty}(\partial \Omega)}\right) \leq C,
$$

for some $C$ independent of $k$. Using the compact embedding $C^{\alpha}(\bar{\Omega}) \hookrightarrow C^{\beta}(\bar{\Omega})$ for $0<\beta<\alpha$, we obtain that there exists another subsequence, that we denote the same, and a function $u^{*} \in$ $C^{\beta}(\bar{\Omega})$ such that $v_{k} \rightarrow u^{*}$ in $C^{\beta}(\bar{\Omega})$. Observe that $v_{k}$ satisfies

$$
\left\{\begin{aligned}
\Delta v_{k}+v_{k} & =0, & & \text { in } \Omega \\
\frac{\partial v_{k}}{\partial n} & =\mu_{k} v_{k}+g\left(\mu_{k}, x, v_{k}\right), & & \text { on } \partial \Omega
\end{aligned}\right.
$$

and the regularity of $g$ implies, $g\left(\mu_{k}, \cdot, v_{k}\right) \rightarrow g\left(\mu^{*}, \cdot, u^{*}\right)$ pointwise. Now hypothesis (H1) and the Lebesgue dominated convergence theorem imply that $g\left(\mu_{k}, \cdot, v_{k}\right) \rightarrow g\left(\mu^{*}, \cdot, u^{*}\right)$ in $L^{r}(\partial \Omega)$ as $k \rightarrow \infty$. 

of

Passing to the limit in the weak formulation of the above equation, we get that $u^{*}$ is a solution

$$
\left\{\begin{aligned}
-\Delta u^{*}+u^{*} & =0, & & \text { in } \Omega \\
\frac{\partial u^{*}}{\partial n} & =\mu^{*} u^{*}+g\left(\lambda^{*}, x, u^{*}\right), & & \text { on } \partial \Omega
\end{aligned}\right.
$$

while the convergence of $v_{k}$ implies $s_{n} \leq s^{*}=P\left(u^{*}\right) \leq s_{n+1}$. Hence, $\left(\mu^{*}, u^{*}\right) \in K_{n}$. This shows the compactness of $K_{n}$.

Observe that since $s_{n}<s_{n}^{\prime}<s_{n+1}$ there exists $(\lambda, u) \in K_{n}$ with $\lambda>\sigma_{1}$. Hence, if we define the number

$$
\lambda_{n}^{*}=\sup \left\{\lambda:(\lambda, u) \in K_{n}\right\} .
$$

then $\lambda_{n}^{*}>\sigma_{1}$ and from the compactness of $K_{n}$ there exists $u_{n}^{*}$ such that $\left(\lambda_{n}^{*}, u_{n}^{*}\right) \in K_{n}$. From i) and the fact that $\lambda_{n}^{*}>\sigma_{1}$, we have that $s_{n}<P\left(u_{n}^{*}\right)<s_{n+1}$. But this implies that there is no solution $(\lambda, u)$ nearby $\left(\lambda_{n}^{*}, u_{n}^{*}\right)$ with $\lambda>\lambda_{n}^{*}$. If this were the case then by continuity of the projection $P$, we would have for such a solution $s_{n}<P(u)<s_{n+1}$ so that $(\lambda, u) \in K_{n}$ and therefore $\lambda_{n}^{*}$ would not satisfy (3.17). Hence $\left(\lambda_{n}^{*}, u_{n}^{*}\right)$ is a supercritical turning point.

With a completely symmetric argument, using the sets

$$
K_{n}^{\prime}=\left\{(\lambda, u) \in \mathcal{D}^{+}, \text {with } P(u)=s, \text { and } s_{n}^{\prime} \leq s \leq s_{n+1}^{\prime}\right\}
$$

and defining $\lambda_{*, n}=\inf \left\{\lambda:(\lambda, u) \in K_{n}^{\prime}\right\}$ we show the existence of $u_{*}$ such that $\left(\lambda_{*, n}, u_{*, n}\right) \in K_{n}^{\prime}$ is a subcritical turning point.

In order to prove the existence of resonant solutions, let us show now the following: there exists $n_{0} \in \mathbb{N}$ large enough such that for each $n \geq n_{0}$ both sets $K_{n}$ and $K_{n}^{\prime}$ contain resonant solutions, that is, solutions of the form $\left(\sigma_{1}, u\right)$.

Let us provide the argument for the sets $K_{n}$. If this is not the case, then there will exist a sequence of integers numbers $n_{j} \rightarrow+\infty$ such that $K_{n_{j}}$ does not contain any resonant solutions. This implies that the compact sets $K_{n_{j}}^{+}=\left\{(\lambda, u) \in K_{n_{j}}: \lambda \geq \sigma_{1}\right\}$ can be written as $K_{n_{j}}^{+}=$ $\mathcal{D}^{+} \cap\left\{(\lambda, u) \in \mathbb{R} \times C(\partial \Omega): \lambda>\sigma_{1}, s_{n_{j}}<P(u)<s_{n_{j}+1}\right\}$ and therefore $K_{n_{j}}^{+}$contains at least a connected component of $\mathcal{D}^{+}$. Moreover it is nonempty since we know that there exists at least one solution $(\lambda, u)$ with $P(u)=s_{n_{j}}^{\prime} \in\left(s_{n_{j}}, s_{n_{j}+1}\right)$ and therefore $\lambda>\sigma_{1}$. The fact that we can construct a sequence of connected componets of $\mathcal{D}^{+}$contradicts the fact that $\mathcal{D}^{+}$is a continuum near $\left(\sigma_{1},+\infty\right) \in \mathbb{R} \times C(\bar{\Omega})$.

A completely symmetric argument can be applied to the sets $K_{n}^{\prime}$. $\square$

With the tools above we can prove now the following.

Corollary 3.5 With the definition of $K_{n}$ and $\lambda_{n}^{*}$ as in the proof of the theorem above (see (3.16) and (3.17)) we can show that, for $n$ large enough,

$$
\left\{\lambda: \lambda \geq \sigma_{1} \text { and } \exists u \text { with }(\lambda, u) \in K_{n}\right\}=\left[\sigma_{1}, \lambda_{n}^{*}\right] .
$$

Similarly, with the definition of $K_{n}^{\prime}$ and $\lambda_{*, n}$, we have

$$
\left\{\lambda: \lambda \leq \sigma_{1} \text { and } \exists u \text { with }(\lambda, u) \in K_{n}^{\prime}\right\}=\left[\lambda_{*, n}, \sigma_{1}\right] .
$$


Proof. Assume the first statement is not true. This means that there exists a sequence of $n_{j} \rightarrow$ $+\infty$ and a number $\tilde{\lambda}_{n_{j}} \in\left[\sigma_{1}, \lambda_{n_{j}}^{*}\right]$ such that there is no function $u \in C(\bar{\Omega})$ with $\left(\tilde{\lambda}_{n_{j}}, u\right) \in K_{n_{j}}$. Since we know that $\left(\lambda_{n_{j}}^{*}, u_{n_{j}}^{*}\right) \in K_{n_{j}}$, then necessarily $\sigma_{1} \leq \tilde{\lambda}_{n_{j}}<\lambda_{n_{j}}^{*}$.

Defining now $\tilde{K}_{n_{j}}=\left\{(\lambda, u) \in K_{n_{j}}, \lambda>\tilde{\lambda}_{n_{j}}\right\}$, then $\tilde{K}_{n_{j}} \neq \emptyset$ since $\left(\lambda_{n_{j}}^{*}, u_{n_{j}}^{*}\right) \in K_{n_{j}}$ and with a similar argument as in the proof of the theorem above, we may show that $K_{n_{j}}$ contains at least a nonempty connected component of $\mathcal{D}^{+}$. The fact that this can be obtained for the whole sequence $n_{j} \rightarrow+\infty$ is in contradiction with the fact that $\mathcal{D}^{+}$is a continuum near $\left(\sigma_{1},+\infty\right) \in$ $\mathbb{R} \times C(\bar{\Omega})$.

A symmetric argument will show the second statement.

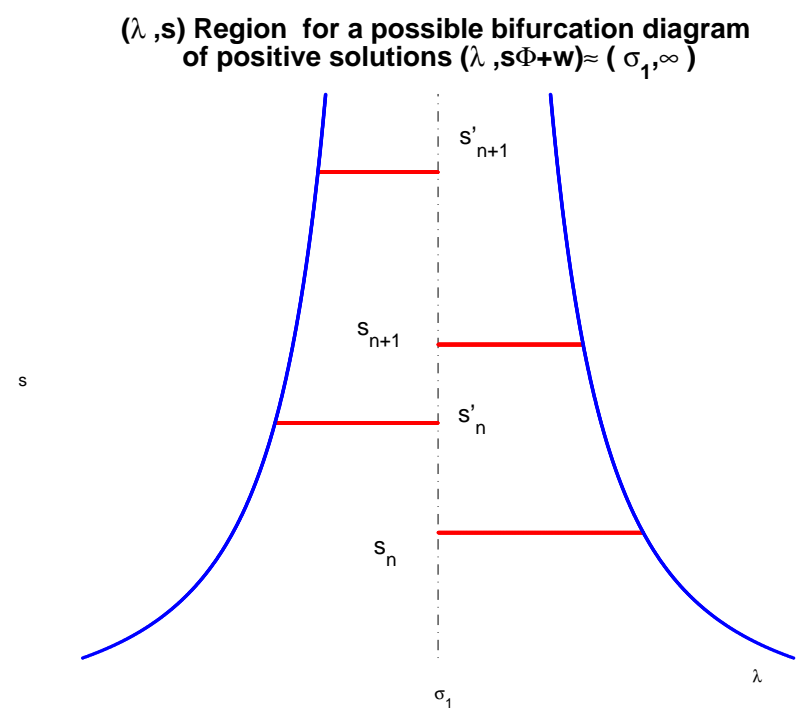

Figure 1: The $(\lambda, s)$ region in $\mathbb{R}^{2}$ for a possible bifurcation diagram is the interior of the solid lines. From part $i$ ) of the Theorem 3.4, the unbounded branch can not cross the solid horizontal lines

\section{Two examples}

\subsection{An oscillatory nonlinearity}

Let us consider an oscillatory nonlinearity of the type (1.4), that is,

$$
g(x, s):=s^{\alpha}\left[\sin \left(\left|\frac{s}{\Phi_{1}(x)}\right|^{\beta}\right)+C\right] \quad \text { with } \alpha<1 .
$$

Applying [1, Theorem 4.3], we have that if $\beta \in \mathbb{R}$ and $C>1$ or if $\beta \leq 0$ and $C>0$ then $\underline{\mathbf{G}}_{+}>0$ and the bifurcation from infinity is subcritical, see (1.3) for a definition of $\underline{\mathbf{G}}_{+}$. 
On the other hand if $\beta \in \mathbb{R}$ and $C<-1$ or if $\beta \leq 0$ and $C<0$ then $\overline{\mathbf{G}}_{+}>0$ and the bifurcation from infinity is supercritical.

Therefore, we consider here the range $\beta>0$ and $-1<C<1$ and note that Theorem 3.4 aplies if

$$
\beta>0, \quad \alpha+\beta<1, \quad \text { and } \quad-1<C<1
$$

Therefore, in this range of parameters, there exist unbounded sequences of subcritical and supercritical solutions, subcritical and supercritical turning points and infinite resonant solutions.

See Figure 2 to visualize the parameter region and a bifurcation diagram.

Remark 4.1 The restriction $\alpha+\beta<1$ on the size of $\beta$ is needed in order to satisfy condition (3.13). This restriction means that although we need "oscillating" nonlinearities $g$, the oscillations cannot be very fast.

At this point it is not clear to us whether this condition is a technical one (as it is suggested by the analysis of the one dimensional problem of the next section) or not. Actually, it may be possible that for higher dimensional problems, some kind of homogenization phenomena may take place for very high oscillating nonlinearities that prevent the formation of turning points and/or resonant solutions.
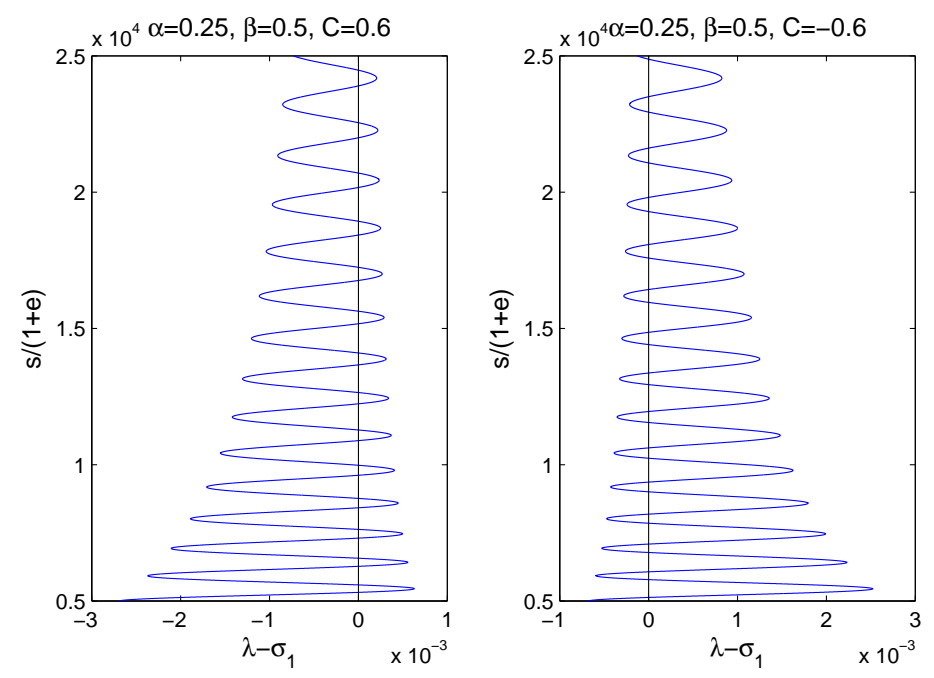

Figure 2: A bifurcation diagram of subcritical and supercritical solutions, containing infinite turning points and infinite resonant solutions.

\subsection{A one dimensional example}

Now we consider the onedimensional version of (1.1), where most computations can be made explicit. 
Observe that equation (1.1) in the one dimensional domain $\Omega=(0,1)$ reads

$$
\left\{\begin{aligned}
-u_{x x}+u & =0, & \text { in }(0,1) \\
-u_{x}(0) & =\lambda u+g(\lambda, 0, u(0)) . & \\
u_{x}(1) & =\lambda u+g(\lambda, 1, u(1)), &
\end{aligned}\right.
$$

The general solution of the differential equation is $u(x)=a e^{x}+b e^{-x}$ and therefore the nonlinear boundary conditions provide two nonlinear equations in terms of two constants $a$ and $b$. The function $u=a e^{x}+b e^{-x}$ is a solution if $(\lambda, a, b)$ satisfy

$$
\left(\begin{array}{cc}
-(1+\lambda) & (1-\lambda) \\
(1-\lambda) e & -(1+\lambda) e^{-1}
\end{array}\right)\left(\begin{array}{l}
a \\
b
\end{array}\right)=\left(\begin{array}{c}
g(\lambda, 0, a+b) \\
g\left(\lambda, 1, a e+b e^{-1}\right)
\end{array}\right)
$$

In this case we only have two Steklov eigenvalues,

$$
\sigma_{1}=\frac{e-1}{e+1}<\sigma_{2}=\frac{1}{\sigma_{1}}=\frac{e+1}{e-1} .
$$

Choosing $g(\lambda, x, u)=g(u)$ and restricting the analysis to symmetric solutions $u_{r}(x)=$ $r\left(e^{x}+e^{1-x}\right)$, with $r \in \mathbb{R}$, it is easy to prove that $u_{r}(x)$ is a solution if and only if $\lambda$ satisfies

$$
\lambda(r)=\sigma_{1}-\frac{g(r(e+1))}{r(e+1)}, \quad r>0 .
$$

Therefore, whenever $g(u)=o(u)$ at infinity, there is an unbounded branch of solutions $\left(\lambda(r), u_{r}\right) \rightarrow\left(\sigma_{1}, \infty\right)$ as $r \rightarrow \infty$.

Fix now

$$
g(u)=u^{\alpha} \sin \left(u^{\beta}\right) \quad \text { for any } \quad \alpha<1, \beta>0 .
$$

From definition (1.3) we can write

$$
\begin{aligned}
& \underline{\mathbf{G}}_{+}=\int_{\partial \Omega} \liminf _{s \rightarrow+\infty} \frac{s g(s)}{|s|^{1+\alpha}} \Phi^{1+\alpha}=\int_{\partial \Omega} \liminf _{s \rightarrow+\infty} \sin \left(s^{\beta}\right) \Phi^{1+\alpha}=-\int_{\partial \Omega} \Phi^{1+\alpha}<0, \\
& \overline{\mathbf{G}}_{+}:=\int_{\partial \Omega} \limsup _{s \rightarrow+\infty} \frac{s g(s)}{|s|^{1+\alpha}} \Phi^{1+\alpha}=\int_{\partial \Omega} \limsup _{s \rightarrow+\infty} \sin \left(s^{\beta}\right) \Phi^{1+\alpha}=\int_{\partial \Omega} \Phi^{1+\alpha}>0
\end{aligned}
$$

and then $\underline{\mathbf{G}}_{+}<0<\overline{\mathbf{G}}_{+}$.

Moreover, by looking in (4.2) at the values of $r \in \mathbb{R}$ such that $\lambda(r)=\sigma_{1}$ we get that $\left(\sigma_{1}, u_{k}\right)$ is a solution for any $k \in \mathbb{Z}$, where

$$
u_{k}(x):=\frac{(k \pi)^{1 / \beta}}{e+1}\left(e^{x}+e^{1-x}\right),
$$

i.e. there is an unbounded sequence of solutions of the resonant problem, see Figure 3.

Moreover, computing in (4.2) the local maxima and minima of $\lambda(r)$ we get that $\left(\lambda_{k}^{*}, u_{k}^{*}\right)$ is an unbounded sequence of turning points, where

$$
\lambda_{k}^{*}:=\sigma_{1}-\frac{(-1)^{k} \alpha}{[(k+1 / 2) \pi]^{1-\alpha}}, \quad u_{k}^{*}(x):=\frac{[(2 k+1) \pi]^{1 / \beta}}{2(e+1)}\left(e^{x}+e^{1-x}\right),
$$

see Figure 3. 

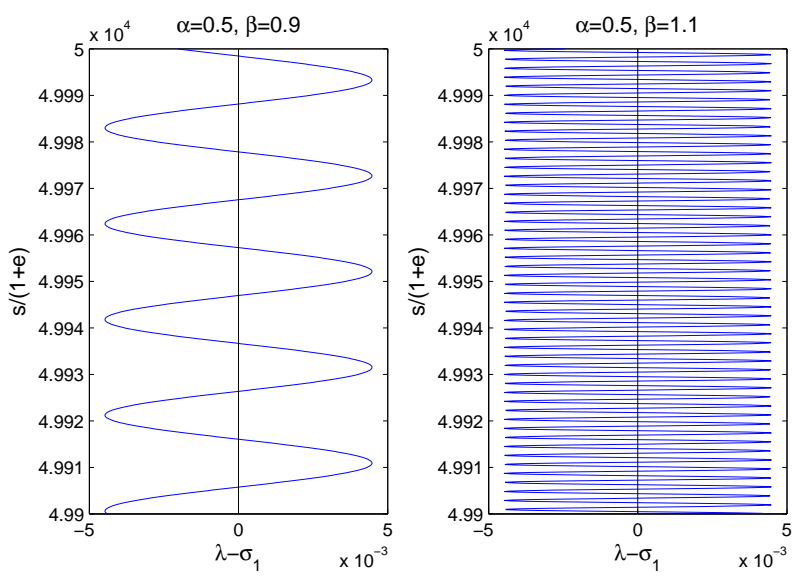

Figure $3: \alpha=0.5, \beta=0.9$ and $\beta=1.1$.

\section{References}

[1] J.M. Arrieta, R. Pardo, A.Rodríguez-Bernal, "Bifurcation and stability of equilibria with asymptotically linear boundary conditions at infinity". Proc. Roy. Soc. of Edinburg, Vol. 137, A, No. 2, 225-252,(2007).

[2] J.M. Arrieta, R. Pardo, A.Rodríguez-Bernal, “Equilibria and global dynamics of a problem with bifurcation from infinity”. Journal of Differential Equations 246, 2055-2080 (2009).

[3] Cañada, A., “ Multiplicity results near the principal eigenvalue for boundary-value problems with periodic nonlinearity", Math. Nachr. 280 (2007), no. 3, 235-241.

[4] E.M. Landesman and A.C. Lazer, Nonlinear Perturbations of linear elliptic problems at resonance, J. Math. Mech., Vol. 19, 609-623, (1970).

[5] P. H. Rabinowitz, "Some global results for nonlinear eigenvalue problems", J. Functional Anal., Vol. 7, 487-513, (1971).

[6] P. H. Rabinowitz, “On Bifurcation From Infinity”, J. Differential Equations, Vol. 14, 462475, (1973). 